\title{
Potenciando la producción oral en inglés: estrategias y herramientas en el aula
}

Enhancing English oral production: classroom strategies and tools

Karina Angélica Mendieta Lira

Universidad César Vallejo - Perú

Lima, Perú

kmendieta@ucvvirtual.edu.pe

\section{RESUMEN}

La expresión oral es una de las habilidades más difíciles de adquirir en el aprendizaje de un idioma extranjero ya que esta se logra cuando el aprendiz está en constante contacto con el idioma. El objetivo de la presente investigación es clasificar y comparar los resultados y hallazgos más importantes sobre las estrategias utilizadas en la producción oral en inglés en investigaciones de los últimos años a nivel nacional e internacional. Para ello, se ha tomado investigaciones publicadas en diferentes revistas indexadas. El resultado obtenido evidencia distintas estrategias y programas de intervención utilizados que han logrado mejorar la producción oral en inglés. Se concluye que estas estrategias dan resultado solo si los docentes están capacitados para hacer uso de ellas y a su vez enriquecer y potenciar el proceso de aprendizaje con el uso de la tecnología.

Palabras Claves: Aprendizaje de inglés; Estrategias de enseñanza; Plataformas virtuales; Producción oral

\section{ABSTRACT}

Oral expression is one of the most difficult skills to acquire in learning a foreign language since it is achieved when the learner is in constant contact with the language. The objective of the present research is to classify and compare the most important results and findings on the strategies used in oral production in English in national and international research in recent years. For this purpose, research published in different indexed journals has been used. The result obtained evidence that different strategies and intervention programs used that have been successful in improving oral production in English. It is concluded that these strategies work only if teachers are trained to make use of them and, in turn, enrich and enhance the learning process with the use of technology.

Keywords: English learning; Oral production; Teaching strategies; Virtual platforms 


\section{Introducción}

En el mundo actual, el idioma inglés es reconocido como la lengua más utilizada en todos los niveles del conocimiento; y es por ello que se ha convertido en un vehículo que permite y facilita la comunicación entre personas de diferentes países y lenguas. En consecuencia, resulta ser uno de los idiomas más predominantes en el mundo, ya que son justamente los países más desarrollados de habla inglesa aquellos que guían el avance científico tecnológico en el conocimiento del hombre (Allegra, M., \& Rodríguez, 2010; García Trejos et al., 2018; Mena, 2014; Muñoz, 2009)time, and spaces to use the language both inside and outside the classroom to interact with others makes the situation even more challenging. This article was conducted as part of the final thesis in the master's degree program in English Didactics from Universidad Surcolombiana, and which aimed at exploring the impact of using Skype as a complementary tool to foster oral production in a group of university students in Neiva, Colombia. Participants included four students from the first level of English with a basic command of the language. Theoretical constructs such the use of Skype in EFL, computer-mediated communication (CMC. Lo que nos da cuenta de cuán importante es el poder comunicarnos en este idioma, ya sea para realizar estudios, investigaciones, trabajo, o simplemente conocer personas de otros países, donde su lengua materna no necesariamente es el inglés, pero que podrán comunicarse gracias a este idioma.

En ese contexto es necesario que se fortalezca la competencia oral, por ello, es imperativo que los docentes desarrollen estrategias adecuadas para mejorar esta, y para lograrlo, el estudiante tiene que estar en constante contacto con el idioma para alcanzar un aprendizaje significativo y las actividades desarrolladas en clase deben estar relacionadas con las preferencias y el contexto del estudiante, porque se ha observado que no basta con lo que aprenden y practican en el aula y es así que se ven con pocas oportunidades de poder practicar el idioma fuera de ella (Pomposo Yanes, 2016; Ramírez Ortíz \& Artunduaga Cuéllas, 2018; Rubio Alcalá, F. D., \& Martínez Lirola, 2008; Yate et al., 2016).

Por otro lado, en el Currículo Nacional del Perú (Minedu, 2016) propone en la educación básica regular dos horas semanales de enseñanza de este idioma con lo cual los estudiantes no obtienen la aptitud necesaria y el manejo de este. Por lo cual no resulta trascendente en la vida estudiantil y profesional de las personas. Además, existen otros problemas que afectan el aprendizaje de una lengua extranjera; como la interferencia que genera la lengua materna en el aprendiz (Alimorad \& Bidoki, 2021; Osborne \& Simonet, 2021), el usar la lengua madre del estudiante en clase ocasiona que éste quiera solo usar estructuras o palabras en su idioma y no en el idioma meta. La ausencia de una buena metodología para la enseñanza de esta lengua (Castro-Crespo et al., 2020), la cual es importante para lograr el objetivo de comunicarse en inglés, y si el docente no la posee no será factible. La motivación con que perciba la lengua el alumno (Keselman \& Yakovleva, 2021)Russia, most of whom are graduates of Orel State University, andwhose teaching experience ranges from 11 to 25 years, es imprescindible que se sienta cómodo en el aula y así poder participar de manera efectiva en el idioma meta. La falta de preparación por parte de los estudiantes en los aspectos generales de la lengua como en su comprensión y producción oral y escrita (Suárez-Cretton et al., 2020), hace que vean el aprender un idioma extranjero como algo muy complicado, porque consideran que no son buenos y es por ello que se ha visto conveniente cambiar la enseñanza de un currículo de contenidos por competencias. 
Asimismo, en el nivel superior en Perú, al igual que en la Educación Básica Regular, los estudiantes solo se encuentran cara a cara con el idioma una vez por semana con dos o tres horas semanales. La Ley Universitaria 30220 (Minedu, 2014) exige egresados competentes, íntegros con conocimientos de las TICS y un idioma extranjero, empero no son suficientes las horas para realmente alcanzar este objetivo. Muchos de los estudiantes optan por estudiar el idioma inglés en un instituto especializado, ya que ven con muy poco nivel sus logros alcanzados en el idioma tanto en la escuela como en la universidad.

La producción oral se fundamenta con la teoría de la Adquisición-Learning de Krashen (Krashen, 1981) la cual, se desarrolló en base a cinco hipótesis, sin embargo, recalca la quinta suposición, en donde afirma que los alumnos que se sienten seguros de sí mismos y están motivados en clase, denotarán mejores resultados en su aprendizaje. Por ello, el autor refirió que hay dos formas de desarrollar la capacidad lingüística, por medio de la adquisición lo cual significa aceptar de manera subconsciente el conocimiento donde la información se almacena y se guarda en el cerebro a través del uso de la comunicación; este es el proceso que se hace referencia para el desarrollo de las lenguas nativas; y el aprender, que comprende lo contrario de adquirir, es la aceptación consciente del conocimiento sobre una lengua, es decir, la gramática o forma.

Este artículo tiene como objetivo clasificar y comparar los diferentes resultados y hallazgos de los trabajos de investigación realizados en los últimos años sobre estrategias para mejorar la producción oral en inglés.

\section{Metodología}

Para realizar el presente trabajo de investigación se hizo una revisión minuciosa de distintos artículos relacionados a la mejora de la producción oral en un idioma extranjero, se tomaron solo publicaciones de los últimos años y han sido recolectadas de revistas indexadas como Scopus, Scielo, Redalyc, Dialnet, entre otras. Se realizó un proceso sistemático para poder obtener las categorías y sub categorías del trabajo (Ver tabla 1).

Tabla 1. Fuentes de revisión

\begin{tabular}{|c|c|c|}
\hline Categorías & Subcategorías & Autores \\
\hline Producción oral & Importancia & Ferro Quintanilla, Daisy Rebeca; Del Pozo Gamarra, Ester Grelte \& Saboya Rios, Nemias. (2017). \\
\hline $\begin{array}{l}\text { Uso de platafor- } \\
\text { mas virtuales }\end{array}$ & $\begin{array}{l}\text { Skype } \\
\text { Langblog y Tan- } \\
\text { dem } \\
\text { Eleclips } \\
\text { Uso de tics } \\
\text { Aprendizaje } \\
\text { Híbrido }\end{array}$ & $\begin{array}{l}\text { García Trejos, Sergio Ramiro; Diaz Pascuas, Rolney Leonardo, \& Artunduaga Cuellar, Marco Tulio. (2018). } \\
\text { Rico Yate, J. P., Ramírez Montoya, M. S., \& Montiel Bautista, S. (2016). } \\
\text { Álvarez Ramos, Eva (2017). } \\
\text { Jordán, Alba; Iza, Sarah \& Parrales, Carmen (2019) } \\
\text { Camargo Pongutá, David. (2018). } \\
\text { Pérez Gómez, Francisco \& Orduz Navarrete, Yehicy. (2018). } \\
\text { Sharma, P., \& Barret, B. (2007) } \\
\text { Sánchez-Narváez, Natalia, \& Chavarro-Vargas, Sergio Alberto. (2017) }\end{array}$ \\
\hline $\begin{array}{l}\text { Inteligencia } \\
\text { Emocional en la } \\
\text { producción oral } \\
\text { de inglés }\end{array}$ & $\begin{array}{l}\text { Clima afectivo } \\
\text { en la producción } \\
\text { oral } \\
\text { Uso de estrate- } \\
\text { gias sociales }\end{array}$ & $\begin{array}{l}\text { Palomino Flores, Paola, \& Almenara, Carlos A. (2019). } \\
\text { Vergara Novoa, Alonso, \& Perdomo Cerquera, María Elena. (2017). } \\
\text { Henríquez Tania, Von Harry, Peña Victor, \& Llanquileo Elena Adriana. (2017). }\end{array}$ \\
\hline $\begin{array}{l}\text { Estrategias } \\
\text { metodológicas } \\
\text { tradicionales }\end{array}$ & $\begin{array}{l}\text { Role play } \\
\text { PBL (aprendizaje } \\
\text { basado en pro- } \\
\text { yectos) } \\
\text { Autoevaluación }\end{array}$ & $\begin{array}{l}\text { Pamplona, J., Cuesta, J.C. y Cano, V. (2019) } \\
\text { Cuitiño Ojeda, Jocelyn, Díaz Larenas, Claudio, \& Otárola, José Luis. (2019). } \\
\text { Reyes Carrasco, Orlando Rubén (2019) } \\
\text { Vaca Torres, A. M., \& Gómez Rodríguez, L. F. (2017). } \\
\text { Ramírez Ortiz, S. M., \& Artunduaga Cuéllar, M. T. (2018). } \\
\text { Barturén Silva, Nancy (2019). } \\
\text { Chalkia, E. (2012). } \\
\text { Caicedo Pereira, Martin Javier; Lozano Bermúdez, Jhonny Alexander, \& Vanegas Medina, Luis Alfonso. (2018). }\end{array}$ \\
\hline
\end{tabular}


A partir de los artículos seleccionados se extrajo el cuerpo de este trabajo, el cual se ha dividido en categorías y subcategorías, para ello, se identificó y analizó los instrumentos y/o estrategias usadas en las intervenciones, así como los resultados obtenidos en cada una de las investigaciones. Para el análisis de estos resultados se tomó en cuenta que fueran resultados positivos y favorables al desarrollo y mejora de la producción oral en un idioma extranjero.

Luego de la revisión y análisis de los resultados de más de 40 investigaciones, se tomaron los aportes de 20 estudios para el desarrollo de este trabajo. Se consideró que estos aportes sean viables y aplicables para estudiantes de todo nivel de educación y de diferentes lenguas madres.

\section{Desarrollo}

\subsection{Producción oral}

La producción oral es la forma que tiene una persona de poder expresar lo que piensa, lo que siente, y se da a través de una serie de estrategias con ciertas pautas a seguir para que la comunicación resulte efectiva (Ferro Quintanilla et al., 2017). Es importante poder transmitir un mensaje claro al receptor y mas aún si es en un idioma extranjero. Por ello, es imprescindible usar todos los recursos que ayuden a tener una expresión oral adecuada y pertinente. La producción oral es una competencia y por tanto requiere de ciertas estrategias comunicativas para llevarse a cabo (Yate et al., 2016). En las sesiones de clase de un idioma extranjero el enfoque que se utiliza es el comunicativo, ya que el objetivo es lograr que los aprendices se comuniquen y/o expresen sus ideas en el idioma meta.

\subsection{Uso de plataformas virtuales en la producción oral}

También Ilamadas entornos virtuales de aprendizaje, tienen como principales funciones las de gestionar, crear y administrar los contenidos propuestos para el aprendizaje haciendo uso de las TICs (Velasteguí, 2019). Hoy en día el uso de plataformas virtuales en especial en la enseñanza superior es bastante común y relevante en la transformación y mejora del proceso de enseñanza aprendizaje. Asimismo, mejora y facilita la comunicación entre docente y estudiante.

\subsubsection{Uso de Skype}

El uso de esta herramienta tecnológica ha permitido no solo la comunicación entre personas de distintos países, sino se ha dado la oportunidad de poder utilizarlo como herramienta de enseñanza, y en este caso del idioma inglés. Esta plataforma ayudó a que los estudiantes que participaron en un estudio de investigación, logren sentirse en confianza y familiarizados al usar el idioma inglés, y a su vez mejoraron su nivel de pronunciación y fluidez (García Trejos et al., 2018)time, and spaces to use the language both inside and outside the classroom to interact with others makes the situation even more challenging. This article was conducted as part of the final thesis in the master's degree program in English Didactics from Universidad Surcolombiana, and which aimed at exploring the impact of using Skype as a complementary tool to foster oral production in a group of university students in Neiva, Colombia. Participants included four students from the first level of English with a basic command of the language. Theoretical constructs such the use of Skype in EFL, computer-mediated communication 
(CMC, ya que vivimos en una época digital en donde es primordial conocer las plataformas que puedan acercarnos a otros de manera remota o virtual. El uso de esta plataforma comunicativa, ha hecho posible que muchos estudiantes puedan mejorar su producción oral y una de las razones del éxito de esta plataforma es que se sienten en confianza y además que están bastante familiarizados con la tecnología.

\subsubsection{Langblog y Tandem}

Son dos plataformas muy utilizadas a nivel mundial para mejorar la producción oral en estudiantes que están adquiriendo una segunda lengua. Se evidenció que el tiempo de conexión y/o permanencia en estas plataformas, ayudó a mejorar el nivel de speaking de los estudiantes (Yate et al., 2016). Estas actividades se desarrollan fuera del horario de clases, como parte de ejercicios adicionales para practicar, ya que el estudiante no tiene mayor contacto con el idioma fuera del aula. Por tanto, el uso y práctica de estas aplicaciones educativas, brindan esos espacios que el estudiante necesita para desarrollar su habilidad de producción oral, y no solo ello, sino que desarrolla también su aprendizaje autónomo, ya que el uso de estas aplicaciones la hace el estudiante y el sistema, no hay un tercer miembro que pueda oírlo, y esto lo hace sentir en confianza de participar libremente, desenvolverse y poder equivocarse sin temor a la crítica y burla, algo que usualmente pasa en las aulas de clase.

\subsubsection{Eleclips}

La creación de esta plataforma se basó en poder mejorar el aprendizaje de un idioma extranjero en entornos virtuales, en donde se complemente tanto la tecnología y la lingüística. Se inclinaron porque la plataforma tenga un enfoque comunicativo, ya que ésta es considerada una de las últimas tendencias pedagógicas, en donde la oralidad debía ser el principal punto de aprendizaje en esta plataforma, y es por ello, que se encuentra dotada de material auditivo real, para que tanto docentes y estudiantes estén en contacto con material de origen nativo (Alvarez Ramos, 2017). A pesar de ser la función comunicativa el principal objetivo de esta plataforma, tiene algunos detalles por mejorar, ya que la producción oral solo se podía dar en un encuentro en la plataforma entre el docente y el estudiante, y éste ha sido el punto débil del uso de la plataforma en aprendizaje de un idioma, puesto que se tenía que concretar el día y hora para conectarse y llevar a cabo la práctica oral, y no siempre podía coincidir docente - estudiante en el mismo horario.

\subsubsection{Uso de las TICs}

El uso de otras herramientas tecnológicas ayuda de manera significativa en la producción oral en inglés. Los estudiantes en el aula desarrollan distintas actividades de práctica oral, pero que muchas veces les resulta aburridas o monótonas y es por ello que se propuso la intervención de un programa con herramientas digitales para promover la participación y a su vez mejorar la expresión oral en el curso de inglés (Jordán et al., 2019). Las TICs siempre serán buenos aliados de los docentes cuando de actividades adicionales se trata, y para ello, es importante que el docente pueda conocer y manejar estas herramientas y poder aplicarlas de manera efectiva dentro del aula. 
Asimismo, se detalla que el uso de la tecnología es de gran utilidad y ayuda para los estudiantes en adquirir una segunda lengua, se enfatiza que los entornos virtuales son necesarios para el desarrollo de las 4 habilidades de los aprendices (Camargo Pongutá, 2018). Dichos entornos virtuales, llámese plataformas para el aprendizaje de un idioma, deben estar diseñados de acuerdo a las preferencias y gustos de los estudiantes, y éste justamente se convierte de alguna manera en un desafío para los docentes para crear el material adecuado en las plataformas o para quienes son los creadores de éstas, que sea agradable y amigable para su proceso de aprendizaje, ya que refieren que de esta manera, cuando es sencillo de entender y resolver actividades, el proceso de enseñanza aprendizaje se torna mucho más viable cuando se considera las necesidades y gustos de los aprendices.

El estudiante no solo debe limitarse a corregir su pronunciación y/o mejorarla dentro de sus horas de clase, sino también debe valerse de materiales externos como el uso de la tecnología a través de diferentes plataformas gratuitas, en donde encontrará ejercicios de expresión oral para repetir e incluso grabarse y reconocer sus errores e ir practicando para mejorar, lo cual se observó que dio resultados positivos (Pérez-gómez \& Orduz Navarrete, 2018). Sin embargo, se resalta la existencia de la interferencia de la lengua materna al momento de producir oralmente en la lengua meta, lo cual ocasiona errores en pronunciación y entonación y es por ello, que sugieren la implementación y uso de recursos tecnológicos fuera del aula para reforzar lo aprendido en el aula, así de esta manera se logrará ver un resultado en los aspectos mencionados líneas arriba.

\subsubsection{Aprendizaje Híbrido o mixto}

Este tipo de aprendizaje es una estrategia que combina elementos y situaciones de una clase presencial con el uso de la tecnología para mejorar el aspecto oral en las sesiones de inglés (Sharma \& Barret, 2007). Su aplicación es importante, ya que no solo se limita al uso del idioma dentro de clases, sino que trasciende fuera de ella. Al aplicar esta estrategia en 7 docentes de una institución educativa, al momento de la intervención utilizaban algunas frases ya conocidas en inglés y también frases nuevas, lo cual se les hacía más sencillo poder transmitir lo que tenían que decir (Sanchez Narvaez \& Chavarro Vargas, 2017) y el estudiante podía captar el mensaje. También demostraron que, en la parte virtual, la herramienta de Skype ayudó mucho para interactuar fuera del aula. Concluyeron que este tipo de aprendizaje, el híbrido o mixto, les da a los estudiantes la oportunidad de mejorar y reforzar el idioma meta adquirido tanto cara a cara como en línea.

\subsection{Inteligencia Emocional}

La inteligencia emocional es referida como la capacidad que tiene un individuo de poder comprender, manejar y utilizar sus propias emociones y también la de otros y es considerada una de las principales competencias que todo profesional debe poseer (Palomino y Almenara, 2019). Es por ello, que esta capacidad sea desarrollada en los estudiantes de segundas lenguas, porque necesitan conocer y controlar sus emociones para poder expresarse en la lengua meta. 


\subsubsection{El clima afectivo}

El ambiente en que se desenvuelve el estudiante es una pieza clave para lograr el objetivo que el estudiante pueda sentirse en confianza y expresarse en la lengua meta. Por ello, propiciar un ambiente adecuado en el aula es fundamental. El ser humano es un ser social y necesita interactuar con otros para poder expresarse y en este caso, poder trabajar en equipo para lograr comunicarse en inglés (Vergara Novoa \& Perdomo Cerquera, 2017). Se sabe que el idioma inglés al igual que el aprendizaje de otras lenguas, trabajan bajo un enfoque comunicativo, se necesita de la interacción de los participantes para poder practicar el idioma, lo cual ha tenido resultados positivos.

Asimismo, se menciona que el filtro afectivo es fundamental en la adquisición de una segunda lengua, si el estudiante no cuenta con 3 factores que se propone que son: la motivación, la autoconfianza y baja ansiedad, podría perjudicar el proceso de aprendizaje. El estudiante tiene que estar predispuesto a querer aprender, de lo contrario, no bastará con solo estar inmerso en un ambiente de la lengua meta.

\subsubsection{Uso de estrategias sociales}

La combinación de las estrategias sociales junto con las estrategias de memoria ayuda a incrementar la producción oral de los estudiantes en el idioma inglés. Para poder aplicar las estrategias sociales, es necesario que el estudiante posea una inteligencia emocional adecuada y a su vez desarrolle la inteligencia interpersonal, ya que aquí deberá interactuar con sus compañeros a través de preguntas, cooperando con algún trabajo en equipo y empatizando con los miembros de su grupo (Henríquez et al., 2017). Por ello, es importante que el docente propicie un ambiente agradable dentro del aula de clases. Estas estrategias, de igual manera, permiten que el estudiante se sienta en confianza, pierda el temor de participar y equivocarse. Asimismo, las estrategias de memoria, ayudarán a incrementar el vocabulario en la lengua meta y ello a su vez permitirá al estudiante tener las palabras necesarias para participar en el grupo de trabajo.

\subsection{Estrategias metodológicas tradicionales en la producción oral}

Las estrategias metodológicas son aquellas que utiliza el docente para lograr y facilitar el contenido de la sesión y que éste sea significativo para los estudiantes y así puedan desarrollar las competencias necesarias para su futuro profesional (Pamplona et al, 2019). Estas estrategias dependerán también del tipo de grupo de estudiantes que se tiene, la cantidad y el ambiente en que se encuentran. Definitivamente las estrategias más utilizadas para el aprendizaje de una lengua extranjera son las comunicativas.

\subsubsection{Role Play}

El role play es definido como la estrategia de dramatización, en donde los estudiantes toman roles distintos asignados por el docente para que interpreten un papel o tarea. Estas tareas asignadas les permitirán a los estudiantes, no solo, desenvolverse mejor en el aula, sino también, mejorar la fluidez y pronunciación en el idioma inglés (Cuitiño Ojeda et al., 2019). Cabe señalar, 
que esta es una estrategia bastante usada, no solo en el aprendizaje de un idioma extranjero, sino en la práctica de diferentes cursos, como el de comunicación que intentan promover la confianza y autocontrol de los estudiantes a través del role play.

En los estudios realizados en donde se aplicó la estrategia del role playing, enfatizan que la aplicación práctica en el proceso de enseñanza-aprendizaje es bastante efectivo dentro del aula; siempre y cuando se priorice y enfatice la participación y producción oral del estudiante a través de actividades reales y vivenciales contextualizadas y/o adaptadas a la realidad de los estudiantes (Reyes, 2019). El estudiante que interprete un rol que se sienta cómodo o le permita expresar emociones o sentimientos, será de mucha utilidad para el progreso en la adquisición de una lengua.

\subsubsection{Aprendizaje basado en proyectos (PBL)}

El aprendizaje basado en tareas o proyectos cumple un rol importante en el aprendizaje de lenguas extranjeras para formar profesionales competentes, donde el docente asigna y orienta distintas actividades a sus estudiantes, como por ejemplo la elaboración de artículos, presentaciones orales, creación de resúmenes, etc.; luego recién realizan el análisis de las estructuras gramaticales en las que los estudiantes hayan podido presentar ciertas dificultades. El uso de esta metodología ayudó a incrementar la competencia en el idioma, esto a su vez, también motivó a los estudiantes a ser quienes solucionen los problemas (Vaca Torres \& Gómez Rodríguez, 2017). Los estudiantes desarrollan una tarea comunicativa utilizando la gramática y vocabulario que han adquirido. Es después que se haya completado las tareas asignadas, que la atención de los estudiantes se enfoca a las formas específicas del idioma.

La aplicación de tareas auténticas, no solo es cualquier trabajo o tarea referente a un tema, sino son tareas de la vida cotidiana que se desarrollan dentro del aula; por ello, los materiales y tareas que se utilizan tienen el objetivo de disminuir cualquier tipo de dificultad que los estudiantes puedan tener al momento de su participación oral, y se puede observar una mejora significativa en la producción oral (Ramírez Ortíz \& Artunduaga Cuéllas, 2018), ya que las tareas están diseñadas también de acuerdo a las necesidades y gustos de los estudiantes, lo cual resulta mucho más sencillo y divertido para ellos hablar sobre esos temas.

Asimismo, se indica que efectivamente el aprendizaje basado en tareas favorece significativamente el desarrollo de la producción oral en los estudiantes (Barturén, 2019). Cuando los estudiantes son expuestos a numerosas presentaciones orales con el fin de trabajar de forma colaborativa y en equipo, esto a su vez, propicia la confianza en sí mismos y en sus compañeros.

\subsubsection{La Autoevaluación}

Es la capacidad que tiene una persona de poder juzgar su propio desempeño y trabajo en alguna actividad encomendada y en base a ello poder tomar decisiones (Chalkia, 2012). Estas decisiones deben ser propuestas de mejora en las actividades dentro de clase. La autoevaluación es muy utilizada en estudiantes universitarios, y usan como herramienta de aprendizaje la grabadora en donde ellos deben grabarse hablando en inglés, usando la gramática y vocabulario correspondiente a la sesión de aprendizaje y luego autoevaluarse, oyendo lo que han dicho y así poder reconocer 
y mejorar sus errores en pronunciación y entonación (Caicedo et al, 2018). Se denota que esta estrategia de evaluación desarrolla también el pensamiento crítico en los estudiantes.

\section{Conclusiones}

Después de haber analizado, clasificado y comparado los diferentes resultados de los trabajos de investigación realizados en los últimos años en diferentes partes del mundo sobre la producción oral en inglés, se puede llegar a las siguientes conclusiones:

La producción oral es una de las habilidades del idioma inglés que más preocupación trae a los docentes, en cuanto a la metodología de enseñanza que deben usar y estrategias a aplicar para, no solo mejorar, sino fomentar en el aula la buena pronunciación, entonación y fluidez en estudiantes de segundas lenguas.

La tecnología es una de las herramientas que más se ha probado en estos estudios y que ha dado resultados favorables, como el uso de Skype, Tandem, Eleclips, etc, ya que estamos viviendo en plena era digital, nuestros estudiantes son nativos digitales, por tanto; se les hace mucho más fácil el uso y manejo de estas plataformas, lo cual ayuda a propiciar la participación activa de los estudiantes e incentiva a usar el idioma meta. De igual manera, se ha observado que puede ser un complemento de la enseñanza que se da de manera presencial, puede llevarlo a la práctica en estas plataformas y le da la oportunidad al estudiante de practicar el idioma en otro ambiente que no sea el aula de clases.

El aspecto emocional y afectivo juega un rol importante en la adquisición de un idioma extranjero, si no se propicia un ambiente agradable y de confianza, el estudiante no sentirá el deseo de participar libremente y tendrá temor de hablar en frente de la clase y cometer errores de pronunciación, lo cual podrá inhibir al estudiante de continuar con sus clases. Por ello, es importante que el docente brinde la confianza necesaria y haga del aula un lugar atractivo y cálido, que fomente la participación activa de todos sus estudiantes.

No se puede dejar de lado la metodología y estrategias tradicionales utilizadas en el aula de clases que, si el docente sabe usarlas de manera correcta, trae muchas ventajas y resultados positivos para los aprendices de segundas lenguas. El juego de roles es una de las estrategias más utilizadas alrededor del mundo y se ha visto la efectividad de este en el aprendizaje del idioma, pero es mucho mejor si se complementa con alguna plataforma que también desarrolla el role play, esto con el objeto de reforzar la producción oral.

Por ello, se sugiere incentivar y utilizar en las sesiones de inglés el uso de la tecnología para enriquecer y potenciar el vocabulario de los estudiantes y a su vez mejorar la producción oral en este idioma. Asimismo, todas las actividades propuestas en las diferentes plataformas virtuales deben considerar temas de acuerdo a la preferencia y características de los estudiantes para hacer de las sesiones de aprendizaje un espacio entretenido y atractivo. 


\section{REFERENCIAS}

Alimorad, Z., \& Bidoki, M. (2021). The effect of using the native language as a pedagogic intervention on iranian EFL learners' complexity of english oral productions. Porta Linguarum: Revista Internacional de Didáctica de Las Lenguas Extranjeras, (36), 83-99. https://doi.org/10.30827/portalin.voi36.15745

Allegra, M., \& Rodríguez, M. (2010). Actividades controladas para el aprendizaje significativo de la destreza de producción oral en inglés como LE. Revista Ciencias de La Educación, (35), 133-152.

Alvarez Ramos, E. (2017). La didáctica de la lengua en entornos virtuales de aprendizaje: el caso concreto de la enseñanza-aprendizaje del español como lengua extranjera y la plataforma Eleclips. RED. Revista de Educación a Distancia, 55(6), 22-34. https://doi.org/10.6018/red/55/6

Camargo Pongutá, D. (2018). Using Information Communication Technologies to Foster the Communicative Competence in Undergraduate Students at the UPTC. Cuadernos de Lingüística Hispánica, 31, 117-135. https://doi.org/10.19053/0121053X.n31.2018.7745

Castro-Crespo, G. C., Garcia-Herrera, D. G., Castro-Salazar, A. Z., \& Erazo-Álvarez, J. C. (2020). Juego de roles: una metodología innovadora para la comprensión lectora. Revista Arbitrada Interdisciplinaria Koinonía, 5(1), 27-46. https://doi.org/10.35381/r.k.v5i1.713

Chalkia, E. (2012). Self-assessment: an alternative method of assessing speaking skills. Research Papers in Langages Teaching and Learning, 3(1), 225-239. https://cutt.ly/kWqjlmi

Cuitiño Ojeda, J., Díaz Larenas, C., \& Otarola, J. L. (2019). Promoción de la fluidez y precisión oral en inglés a través del role play. Cuadernos de Investigación Educativa, 10(1), 43-62. https://doi.org/10.18861/ cied.2019.10.1.2880

Ferro Quintanilla, D. R., Del Pozo Gamarra, E. G., \& Saboya Ríos, N. (2017). Programa "Oral expression in the classroom" para el desarrollo de la expresión y comprensión oral del idioma inglés. Apuntes Universitarios, 7(2), 2-11. https://doi.org/10.17162/au.v7i2.191

García Trejos, S. R., Diaz Pascuas, R. L., \& Artunduaga Cuellar, M. T. (2018). Las sesiones de Skype como una forma de proporcionar práctica oral adicional a los estudiantes universitarios de inglés TT Skype sessions as a way to provide additional oral practice of English university students. Colombian Applied Linguistics Journal, 20(1), 62-78. https://doi.org/10.14483/22487085.10826

Henríquez, T. K., Von Johnn, H. I., Peña, V. H., \& Llanquileo, E. A. (2017). The Effects of Memory and Social Strategies on Oral Production. Colombian Applied Linguistics Journal, 19(2), 209. https://doi. org/10.14483/22487085.10535

Jordán, A. R., Iza, S. J., \& Parrales, C. S. (2019). Interactive methodological strategy in the English language for the development of oral expression and comprehension. Espacios, 40(26).

Keselman, I., \& Yakovleva, Y. (2021). Short teacher responses in the EFL classroom: A corpus-approach assessment. Journal of Language and Education, 7(2), 175-188. https://doi.org/10.17323/JLE.2021.9767

Krashen, S. (1981). Second Language Acquisition and Second Language Learning. Oxford: Pergamon Press.

Mena, M. I. S. (2014). Propuesta de enseñanza basada en el modelo speaking: desarrollo de la producción oral. Amazonia Investiga, 3(4), 145-165. https://www.amazoniainvestiga.info/index.php/amazonia/ article/view/665

Minedu. (2014). Ley Universitaria 30220 - Ministerio de Educación 2014.

Minedu. (2016). Currículo Nacional - Ministerio de Educación. MINEDU.

Muñoz, D. (2009). Estrategias de Aprendizaje para el desarrollo de la producción oral en la Licenciatura de Lenguas Modernas de la Pontificia Universidad Javeriana. [tesis de grado, Pontificia Universidad Javeriana] Repostorio Institucional http://hdl.handle.net/10554/5855

Osborne, D. M., \& Simonet, M. (2021). Foreign-Language Phonetic Development Leads to First-Language Phonetic Drift: Plosive Consonants in Native Portuguese Speakers Learning English as a Foreign Language in Brazil. Languages, 6(3), 112. https://doi.org/10.3390/LANGUAGES6030112 
Pérez-gómez, F. A., \& Orduz Navarrete, Y. (2018). La interferencia en ejercicios de producción oral de docentes en formación de tercer semestre de inglés como lengua extranjera. Trabalhos Em Linguística Aplicada, 57(2), 1043-1078. https://doi.org/10.1590/010318138650123302391

Pomposo Yanes, M. L. (2016). La evaluación de la competencia oral en segundas lenguas. El caso del inglés en el mundo profesional. Didáctica. Lengua y Literatura, 28(0), 243-262. https://doi.org/10.5209/ dida.54091

Ramírez Ortiz, S. M., \& Artunduaga Cuéllar, M. T. (2018). Authentic Tasks to Foster Oral Production Among English as a Foreign Language Learners. HOW Journal, 25(1), 51-68. https://doi.org/10.19183/ how.25.1.362

Rubio Alcalá, F. D., \& Martínez Lirola, M. (2008). La comunicación oral del inglés en España: influencia de los aspectos educativos y mediáticos en el éxito del aprendizaje. Revista de Enseñanza Universitaria, $32,51-63$.

Sanchez Narvaez, N., \& Chavarro Vargas, S. A. (2017). Efl Oral Skills Behavior When Implementing Blended Learning in a Content-Subject Teachers'1 Professional Development Course. Colombian Applied Linguistics Journal, 19(2), 263. https://doi.org/10.14483/22487085.11964

Sharma, P., \& Barret, B. (2007). Aprendizaje mixto: Uso de la tecnología dentro y fuera del aula de idiomas. MacMillan Education.

Suárez-Cretton, X., Castro-Méndez, N., \& Muñoz-Vilches, C. G. (2020). Uso de juego de roles con grabación de video para el desarrollo de la competencia de entrevistar en estudiantes de psicología. Revista Electrónica Educare, 24(2), 1-18. https://doi.org/10.15359/ree.24-2.2

Vaca Torres, A. M., \& Gómez Rodríguez, L. F. (2017). Increasing EFL Learners' Oral Production at a Public School Through Project-Based Learning. PROFILE Issues in Teachers' Professional Development, 19(2), 57-71. https://doi.org/10.15446/profile.v19n2.59889

Velasteguí, P. (2019). Plataformas virtuales y su impacto en la Educación Superior. Explorador Digital, 1(4), 5-21. https://doi.org/10.33262/exploradordigital.v1i2.318

Vergara Novoa, A., \& Perdomo Cerquera, M. E. (2017). Fortalecimiento de la expresión oral y escrita en inglés a través de un andamiaje de escritura creativa colaborativa: un estudio de diseño desde la cognición distribuida. Forma y Función, 30(1), 117. https://doi.org/10.15446/fyf.v3on1.62417

Yate, J. P. R., Montoya, M. S. R., \& Bautista, S. M. (2016). Desarrollo de la competencia oral del inglés mediante recursos educativos abiertos. Apertura, 8(1), 1-15. http://www.redalyc.org/articulo. oa?id $=68845366006$

\section{AUTORA}

Karina Mendieta Lira. Magíster en Educación con mención en docencia y gestión educativa de la Universidad César Vallejo en Lima, Perú. Licenciada en Ciencias de la Educación de la Universidad Nacional de Educación Enrique Guzmán y Valle. Docente de inglés en el nivel superior, Coordinadora Académica del área de inglés. Líneas de investigación: Evaluación y aprendizaje en idioma extranjero.

\section{Conflicto de intereses}

Karina Angélica Mendieta Lira informa que no existe conflicto de interés posible.

\section{Financiamiento}

No existió asistencia financiera de partes externas al presente artículo.

\section{Agradecimientos}

Gracias a Dios por todas las bendiciones y a mis padres por el soporte que me dan cada día y son el motivo por el cual seguir adelante. 
